\title{
Investigation of Weldability of Multi-layer Metals by Laser Impact Welding
}

\author{
Shuai GAO ${ }^{a,{ }^{*}}$, Hui-Xia LIU ${ }^{b}$, Zong-Bao SHEN ${ }^{c}$, Xiao WANG ${ }^{d}$, and You-Juan \\ $\mathrm{MA}^{\mathrm{e}}$
}

School of Mechanical Engineering, Jiangsu University, Zhenjiang, Jiangsu 212013, China

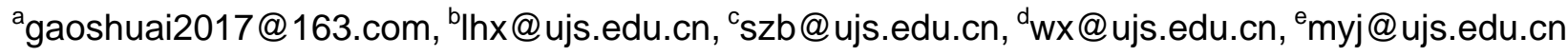

* Shuai GAO

Keywords: Laser impact welding (LIW), Three layer aluminum foils, Interface morphology, Weldability.

\begin{abstract}
Laser impact welding is a relatively novel solid welding technique. In this study, weldability of three layer aluminum foils by laser impact welding has been experimentally investigated. Besides, the welding area and interface morphology of the welded joints were also studied. Experiment results show that three layer aluminum foils can be bonded by laser impact welding. A circular welding area was observed in welded joint. Metallograpic investigation indicates that the joint interface between flyer plate and intermediate plate was flat. The bonding interface between intermediate plate and base plate was transformed from flat to wave, which is similar to electromagnetic pulse welding. Therefore, it is a promising welding method in the welding of multi-layer metal foils.
\end{abstract}

\section{Introduction}

High speed impact welding (HSIW) is a solid state, collision-based bonding technique, and is well known for its capability to directly join a wide variety of both similar and dissimilar combinations of metals with large difference in melting points, thermal expansion coefficient which are difficult to be welded by fusion welding. Furthermore, the formation of intermetallic phase and the heated affected zone can be minimized by means of HSIW [1,2]. The rapid development of aerospace, automobile, chemical industry, shipbuilding and other industries and the demand of dissimilar material joining also promote the development of HSIW. Generally, there are two kinds of impact welding methods, namely explosive welding (EXW) [3] and magnetic pulse welding (MPW) [4]. They share similar impact-driven solid state welding principle, but have different driving force. Therefore, they are often applied in different fields. EXW is suited for joining similar and dissimilar metals for large planar plates [5,6]. MPW is focus on tube welding [7,8].

In recent years, joining of metal foil has wide applications for micro device manufacturing [9]. In order to meet industry demand, laser impact welding was developed [10], which utilizes laserinduced shock wave to accelerate the flyer plate towards the base plate to obtain the metallurgical bonding. One remarkable feature of this method is that it is suitable for small foil thickness and length scales, and does not depend on the electrical conductivity of the flyer plate [11]. Consequently, LIW technology has received great attention with its unique advantages. Zhang et al. [11] welded Al /Al and $\mathrm{Al} / \mathrm{Fe}$ through LIW. Wang et al. [12,13] studied laser shock welding of $\mathrm{Al} / \mathrm{Al}, \mathrm{Al} / \mathrm{Cu}$ and $\mathrm{Al} / \mathrm{Ti}$. Wang et al. [9] focused on the design of apparatus and parametric optimization. More recently, Wang et al. [14] presented laser impact spot welding through experimental and numerical study.

To the best of our knowledge, there is no report about LIW of multi-layer metal foils. Joining of multi-layer metal foils by LIW has a broad prospect for the manufacture and assembly of micro-devices. Additionally, there may be some differences in bonding process between welding of multi-layer metal foils and two layer metal foils. Therefore, it is interest to investigate the weldability of LIW of multi-layer metal foils. In this study, three layer pure aluminum foils were welded by LIW under different laser energy. Then, bonding area and interface morphology was observed by 
metallographic investigation on cross-sections of joint areas.

\section{Principal and Experimental Design}

The basic concept of laser impact welding of three layer metal foils is illustrated in Fig. 1. The experimental apparatus mainly consists of Nd: YAG laser, confinement layer, ablative layer, flyer plate, intermediate plate, base plate, filler piece, back support, and clamp. As shown in Fig. 2(a), when a intense laser pulse irradiates the ablative layer, which absorbs laser energy and vaporizes into plasma. Confined by the confinement layer, the plasma pushs the flyer plate towards the intermediate plate with high speed as shown in Fig. 2(b). Upon collision, metallurgical bonding occurs with proper collision angle [11] as shown in Fig. 2(c). Then, the bonded flyer plate and intermediate plate moves toward the base plate, and welding happens under proper impact velocity and impact angle as shown in Fig. 2(d).

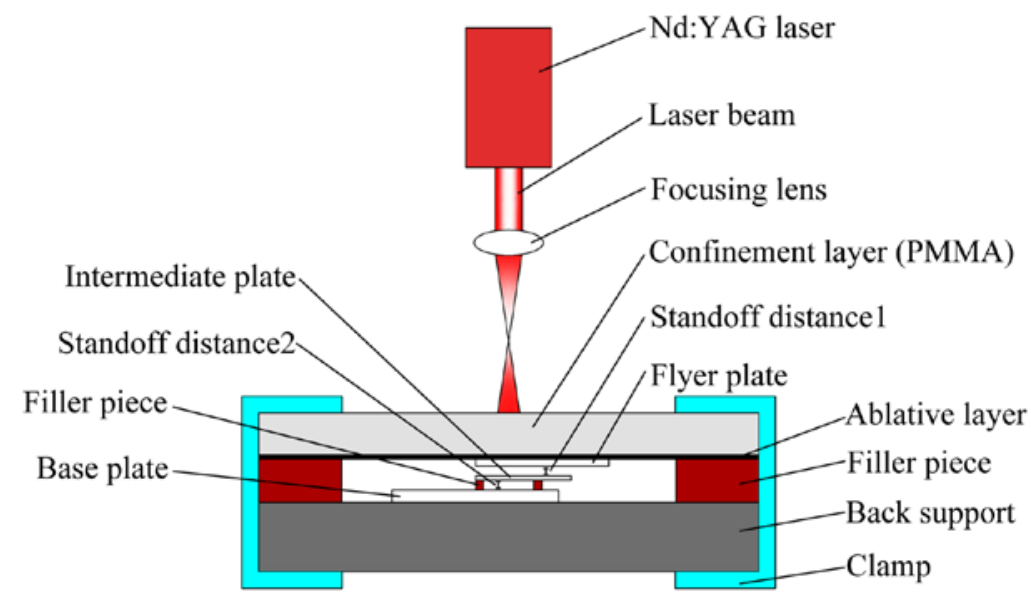

Fig. 1 Schematic diagram of laser impact welding of three-layer metal foils
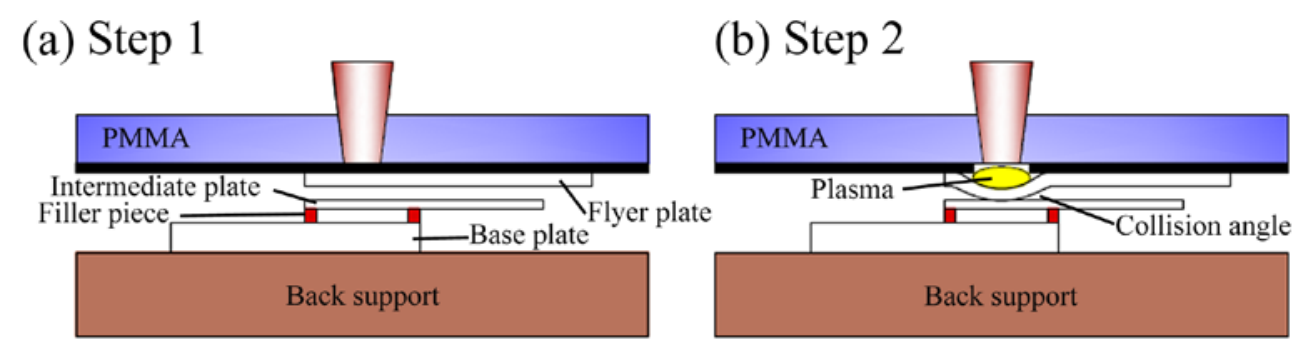

(c) Step 3
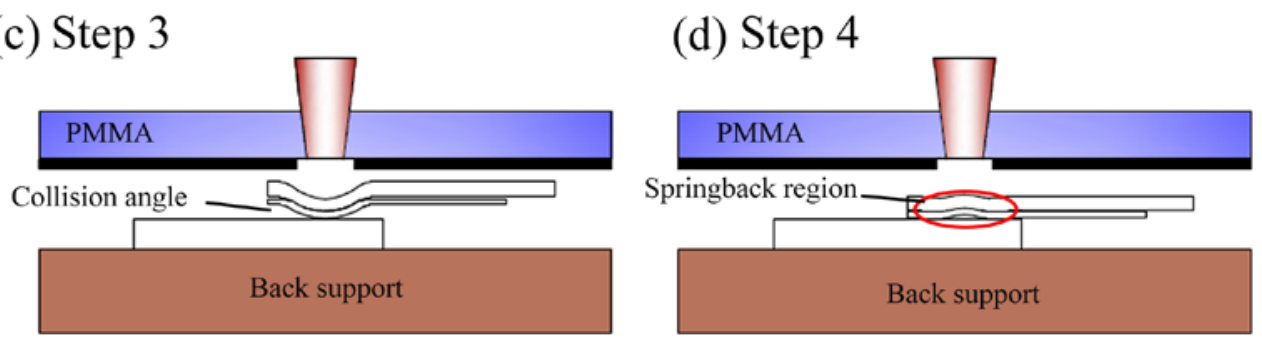

Fig. 2 Schematic illustration of deformation process during laser impact welding

In the experiment, Al1060 sheets with the thickness of $0.2 \mathrm{~mm}, 0.02 \mathrm{~mm}$ and $0.08 \mathrm{~mm}$ were selected as the base plate , the intermediate plate and the flyer plate respectively. The base plate was cut for the dimension of $60 \mathrm{~mm} \times 30 \mathrm{~mm}$. The intermediate plate was cut for the dimension of $25 \mathrm{~mm} \times 8 \mathrm{~mm}$ and 
the flyer plate was cut for the dimension of 30mm x 8mm. A PMMA layer with the thickness of 3.6mm was selected as the confinement layer. A thin layer of black lacquer was chosen as the ablative layer and painted on the PMMA. The flyer palte was stuck to the ablative layer by the Loctite 380 . The imtermediate plate was fixed on the filler piece. The base plate was fixed on the back support. The standoff distance was adjusted by changing the thickness of filler piece. The laser used in this experiment was the Nd YAG-GAIA R laser with Gaussian distribution beam. The range of pulse energy is 2J-6J, and the wavelength is $1064 \mathrm{~nm}$. Moreover, the pulse duration is about 20ns. In present study, 3mm diameter laser spot size was applied. The values of standoff 1 and standoff 2 were $0.4 \mathrm{~mm}$ and $0.3 \mathrm{~mm}$ respectively, and four kinds of laser energy were used. The detail parameters were listed in Table 1.

Table 1 Detail experimental parameters

\begin{tabular}{llllllll}
\hline Parameters & $\begin{array}{l}\text { Flyer } \\
\text { plates } \\
{[\mathrm{mm}]}\end{array}$ & $\begin{array}{l}\text { Intermediate } \\
\text { plates [mm] }\end{array}$ & $\begin{array}{l}\text { Base } \\
\text { plates } \\
{[\mathrm{mm}]}\end{array}$ & $\begin{array}{l}\text { Standoff } \\
\text { distance1 } \\
{[\mathrm{mm}]}\end{array}$ & $\begin{array}{l}\text { Standoff } \\
\text { distance2 } \\
{[\mathrm{mm}]}\end{array}$ & $\begin{array}{l}\text { Laser spot } \\
\text { size[mm] }\end{array}$ & $\begin{array}{l}\text { Laser } \\
\text { energy[J] }\end{array}$ \\
\hline Value & $30 \times 8 \times 0.08$ & $25 \times 8 \times 0.02$ & $60 \times 30 \times 0.2$ & 0.4 & 0.3 & 3 & $\begin{array}{l}3.2,3.6,4.0, \\
4.4\end{array}$ \\
\hline
\end{tabular}

\section{Results and Discussions}

Three layer aluminum foils plates were welded and when the laser energy was 3.2J, bonding could not be achieved. Because of an insufficient laser energy, a high-temperature and high-pressure plasma induced by a pulsed and intense laser pulse was not enough to accelerate the flyer plate. Consequently, sufficient pressure cannot be produced. Thus, bonding was not obtained. However, the other laser energy led to the required collision pressure and bonding could be obtained. As shown in Fig. 3, a spot-like welding of about $2.95 \mathrm{~mm}$ was achieved when 3.6J laser energy and 3mm diameter laser spot was applied. Moreover, the collision region of flyer plate maintains high surface quality with little plastic deformation.

In order to observe the welding area and interface morphology of the two bonding interface, the measuring system of true color confocal microscope for materials microscopy with scanning stage (Axio CSM 700) was utilized for metallographic analysis for cross-sections of the weld joints.

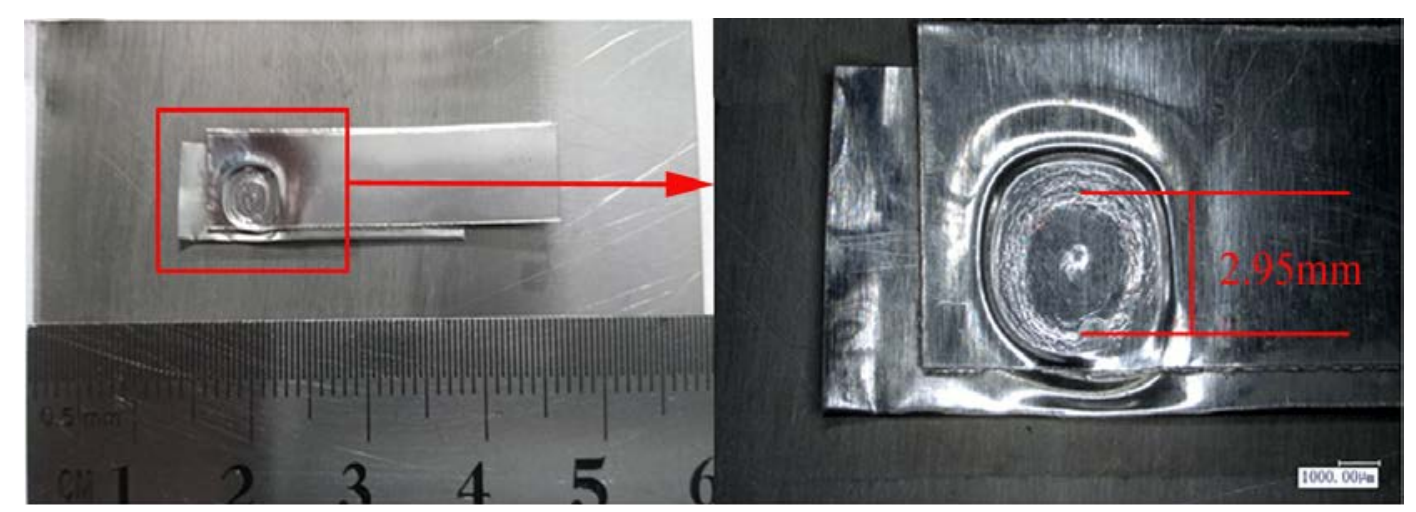

Fig. 3 Weld example when laser energy was 3.6J

Weld area. The welding area is one of the important factors of welding quality. In particular, welding area has great effect on the joint strength. As shown in Fig. 4, the bonding zone is circular in laser impact welding of three layer Al1060 foils due to springback of the central region. Similar results were received in LIW of two layer metal plates [14]. It is probaly because that the impact angle below the critical value and bonding does not happen in central region. Methods to increase the welding area should be presented through further theoretically and practically investigation to improve the mechanical properties of joints. 


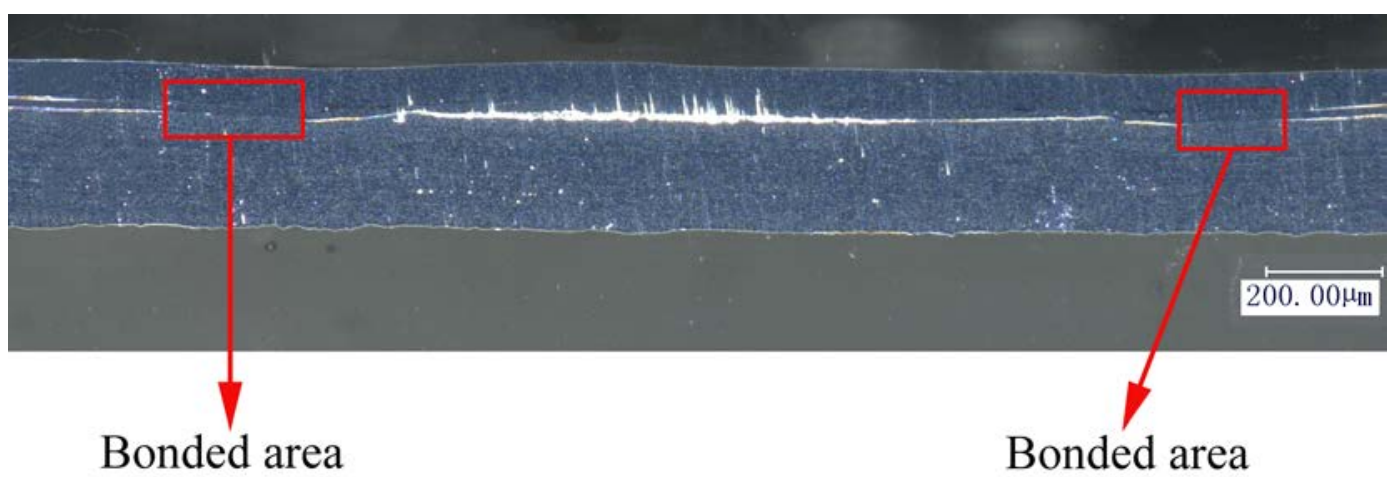

Fig. 4 Optical micrographs of the cross sectional view of $\mathrm{Al} / \mathrm{Al} / \mathrm{Al}$ spot joint (laser energy :4J)

Weld interface. Characteristics of welding interface has always been a research hotpot in HVIW [5,11-14]. Normally, there are three kinds of interface morphology of HVIW including flat interface, wave interface and wave pattern with vortex shedding. Moreover, both flat interface and wave inteface with small waves were obtained by LIW [11-14], and it may account for this phenomenon that the impact velocity and flyer plate thickness for LIW is relatively lower compared with EXW and MPW.

A flat interface was observed between flyer plate and intermediate plate as shown in Fig. 5. When high velocity collision happens between flyer plate and intermediate plate, bulging of intermediate plate happens along with the bonding process. The loss of the kinetic energy from flyer plate is small. Thus, plastic deformation is also small in the collision zone. As shown in Fig. 5, along with the collision point moves from the center toward the left along the radius, the welding interface between intermediate plate and base plate also experiences a smooth transition from straight to wave, which is similar to electromagnetic pulse welding $[7,8]$. When collision point moves from the center toward the left, impact angle also gradually increases then decreases. Upon the impact angle reach a threshold, the wave appeared [14]. Moreover, there was not heat affected zone and intermetallic phase zone near the interface, and Zhang et al [11] and Wang et al [9] also found this phenomenon. That was probably because that interface temperature produced by heavy shear deformation is below the melting temperature of plates.
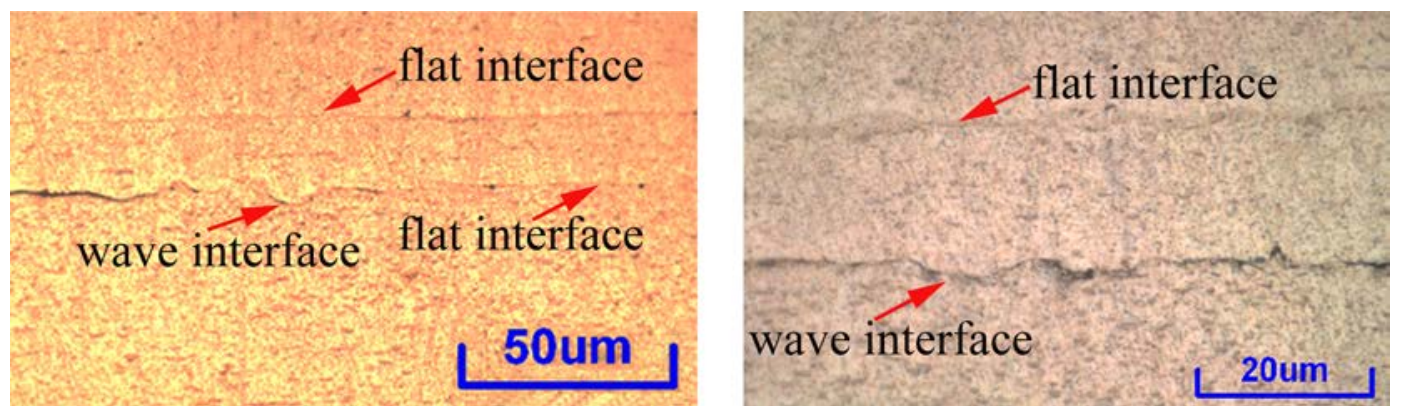

Fig. 5 Optical micrographs of the local amplification view for the weld interface Al/Al/Al joint(left side)

\section{Summary}

The purpose of this paper is to investigate the multi-layer metal foils weldability by LIW. Three layer aluminum foils were welded under four kinds of laser energy. Besides, the welding area and interface morphology of the welded joints were also studied by metallographic investigation. The following conclusions can be made based on this study. 
(1) Three layer aluminum foils can be welded by using LIW under appropriate weld parameters.

(2) Welding area is circular.

(3) Welding interface is flat between flyer plate and intermediate plate. The bonding interface between intermediate plate and base plate was transformed from flat to wave, which is similar to electromagnetic pulse welding.

However, this paper only studies the feasibility of laser impact welding of three layer aluminum foil plates. The effect of the process parameters including laser energy, laser spot size and standoff distance on interface microstructure and mechanical properties should be further studied to obtain better welding joints. The bongding mechanism should be deeply investigated.

\section{Acknowledgments}

This work is supported by the National Natural Science Foundation of China (No.51175235), the Natural Science Foundation of Jiangsu province (No. BK20151343.), the scientific research project of Jiangsu University (No.14A115) and the college students practice innovation fund of industry center (No. ZXJG201587).

\section{References}

[1] T.A. Palmer, J.W. Elmer, D. Brasher, D. Butler, R. Riddle, Development of an explosive welding process for producing high-strength welds between niobium and 6061-T651 aluminum, Weld J -New York, 85 (2006) 252-263.

[2] A. Ben-Artzy, A. Stern, N. Frage, V. Shribman, Interface phenomena in aluminium magnesium magnetic pulse welding, Sci Technol Weld Join. 13 (2008) 402-408.

[3] F. Findik, Recent developments in explosive welding, Material and Design. 32 (2011) 1081-1093.

[4] A. Kapil, A. Sharma, Magnetic pulse welding: an efficient and environmentally friendly multi-material joining technique, Journal of Cleaner Production. 100 (2015) 35-58.

[5] N, Zhang, W.X. Wang, X.Q. Cao, J.Q. Wu, The effect of annealing on the interface microstructure and mechanical characteristics of AZ31B/AA6061 composite plates fabricated by explosive welding, Materials and Design. 65 (2015) 1100-1109.

[6] C. Borchers, M. Lenz, M. Deutges, H. Klein, F. Gärtner, M. Hammerschmidt, H. Kreye, Microstructure and mechanical properties of medium-carbon steel bonded on low-carbon steel by explosive welding, Materials and Design. 89 (2016) 369-376.

[7] V. Psyk, D. Risch, B.L. Kinsey, A.E. Tekkaya, M. Kleiner, Electromagnetic forming-A review, Journal of Materials Processing Technology. 211 (2011) 787-829.

[8] R. Raoelison, N. Buiron, M. Rachik, D. Haye, G. Franz, M. Habak, Study of the elaboration of a practical weldability window in magnetic pulse welding, Journal of Materials Processin Technology. 213 (2013) 1348-1354.

[9] H. M. Wang, G. Taber, D.J. Liu, S. Hansen, E. Chowdhury, S. Terry, J. C. Lippold , G. S. Daehn, Laser impact welding: Design of apparatus and parametric optimization ,Journal of Manufacturing Processes. 19 (2015) 118-124.

[10] G.S. Daehn, L. JC: US Patent PCT/US09/36299 (2009).

[11] Y. Zhang, S.S. Babu, C. Prothe, M. Blakely, J. Kwasegroch, M. LaHa, G.S. Daehn, Application of high velocity impact welding at varied different length scales, Journal of Materials Processing Technology. 211 (2011) 944-952. 
[12] X. Wang, C.X. Gu, Y.Y. Zheng, Z.B. Shen, H.X. Liu, Laser shock welding of aluminum/ aluminum and aluminum/copper plates, Materials and Design. (2014) 26-30.

[13] Y.X. Gu, X. Wang, E.R. Hao, Y.Y. Zheng, T.B. Qiu, Y.J. Ma, H.X. Liu, Experimental study on laser impact welding of dissimilar metals, Key Engineering Materials. 621 (2014) 19-24.

[14] X. Wang, Y.X. Gu, T.B. Qiu, Y.Y. Ma, D. Zhang, H.X. Liu, An experimental and numerical study of laser impact spot welding. Materials and Design, 65 (2015) 1143-1152. 